(C2015 IEEE. Personal use of this material is permitted. Permission from IEEE must be obtained for all other uses, in any current or future media, including reprinting/republishing this material for advertising or promotional purposes, creating new collective works, for resale or redistribution to servers or lists, or reuse of any copyrighted component of this work in other works. DOI: ¡10.1109/MMS.2015.7375430; 


\title{
SAR Imaging in an Automotive Scenario
}

\author{
Hasan Iqbal, Muhammad Babar Sajjad, Michael Mueller, and Christian Waldschmidt \\ Institute of Microwave Engineering \\ University of Ulm \\ Ulm, Germany \\ Email: Hasan.Iqbal@Uni-Ulm.de
}

\begin{abstract}
In this paper a $77 \mathrm{GHz}$ patch array radar is used in combination with the line processing and range Doppler algorithms to produce synthetic aperture radar (SAR) images of automotive scenarios. Measurements are made using a linear rail unit and the images are briefly discussed.

In the latter part of the paper, a position error investigation is carried out, where the measurements are customised to fit a real automotive scenario where the automobile experiences acceleration errors which are not detected by the acceleration sensors fixed in the car. The images with errors are then presented and a quantitative analysis is carried out.
\end{abstract}

\section{INTRODUCTION}

Recent years have witnessed tremendous growth in the automotive safety and driver assistance sector. A crucial component of any automotive safety system is a radar, which enables the automobile to 'see'. Conventional automotive radar systems mostly look forward in the direction of travel since that is the area of greatest interest to a driver. Keeping in mind, however, the progress being made towards autonomous automobiles [1] [2], it is arguably equally crucial to have a radar which is capable of imaging the scenario to the side of the automobile, so that sensitive road users and traffic situations can be recognised in good time, without the assistance of a human driver.

The requirement of an imaging radar can be fulfilled by synthetic aperture radar (SAR), which can take images of the stationary objects in the scenario next to the car as the car is being driven. Though efforts have previously been made to implement SAR for automotive applications, they have either focused mainly on simulations [3] or the experimental implementations [4], [5] have mostly focused on parking lot detection and less on providing high resolution radar images where automobiles or other objects present in the traffic scenario can be identified. In this paper, results are presented of SAR measurements of stationary objects in an automotive scenario, obtained using two different SAR algorithms and an in-house developed patch array radar system.

\section{Measurement Setup and Algorithm}

The radar used for the measurement setup was developed at the institute [6]. It has 32 transmit and 2 receive antennas, however, for the purpose of SAR measurements, only a single transmit and receive antenna were used. The bandwidth used for the measurements was $5 \mathrm{GHz}$, which amounts to a theoretical range resolution of roughly $3 \mathrm{~cm}$.

This radar was mounted on a linear rail unit and it was made to traverse $4 \mathrm{~m}$ parallel to the scene of interest which was to be imaged. The rail unit moves with the help of a stepper motor and it was programmed such that radar snapshots were taken with a step distance of $0.5 \mathrm{~mm}$ for the length of $4 \mathrm{~m}$. This corresponds to a total of 8000 radar measurements, which cover the whole scene of interest.

The measurement setup is shown in Fig. 1 with the scene of interest being the two cars parked in front of the setup. The separation of the cars is $60 \mathrm{~cm}$. The SAR image for this configuration is shown in Fig. 2. Similar setups were also used to image a single car from various perspectives, from the front, rear and side. Figure 3 shows a SAR image of a car imaged from the front. The results are discussed in depth in the next section.

The two SAR algorithms implemented and compared in this paper are the range Doppler [7] and the line processing algorithm [8]. The algorithms function by defining a certain synthetic aperture length $\left(L_{\text {syn }}\right)$ which would give a specific azimuth resolution. This length is dependent on the physical size of the aperture, the wavelength of operation and the range at which a particular target is situated. For the line processing algorithm, there is a direct relationship between the quality of the image and $L_{\mathrm{syn}}$. This is illustrated in the plot shown in Fig. 4 where the normalised power is plotted for various synthetic aperture lengths for a single range bin (at $1.8 \mathrm{~m}$ ) over the entire azimuth length for the same radar image shown in Fig. 3. It can clearly be seen that a longer $L_{\mathrm{syn}}$ results in better power performance.

Choosing different $L_{\text {syn }}$ for the range Doppler algorithm (RDA) does not affect the normalised power as strongly. This is due to the way the RDA functions, where the processing is done in frequency mode and the image data for one $L_{\mathrm{syn}}$ obtained by an inverse FFT. Then to obtain the complete image, all the image data for individual $L_{\text {syn }}$ are summed, thus pushing up the power levels across the image.

The win in azimuth resolution does not always justify the need for a longer aperture. Additionally, as will be seen later, a longer $L_{\text {syn }}$ leads to greater integration of an acceleration error. Hence a compromise was reached and in this paper all results presented are for an $L_{\mathrm{syn}}$ of $1 \mathrm{~m}$.

\section{RESULTS}

After the measurements were carried out as described in the earlier section, the results were fed into the two SAR processing algorithms and the radar images thus obtained are 


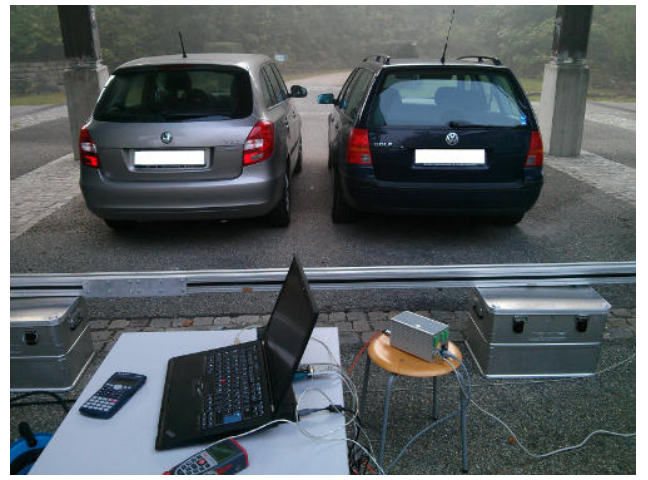

Fig. 1. Measurement setup.

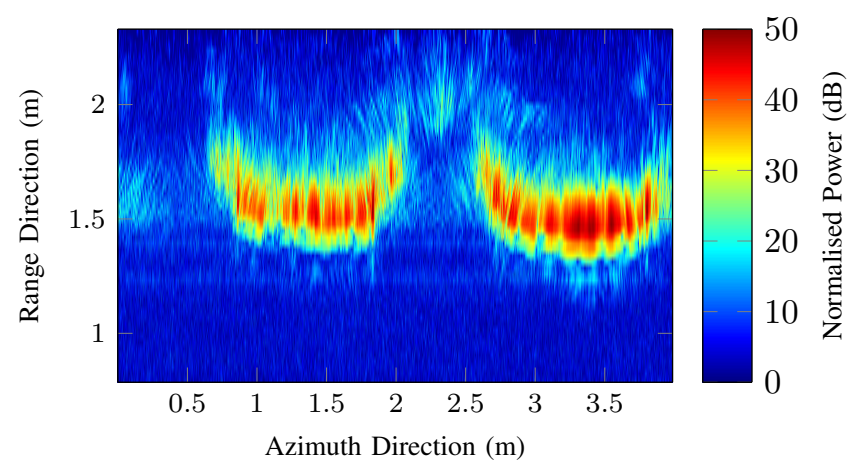

Fig. 2. Measurement result of two cars rear view.

discussed in this section. Shown in Fig. 2 is the radar image corresponding to the measurement scenario illustrated in Fig. 1 in the previous section. The result is a high resolution radar image of the two cars. The contour of the two cars can clearly be made out through the grouping of the various scattering centres [9]. The two cars were parked deliberately so close $(60 \mathrm{~cm})$ together to make the scenario slightly challenging for the algorithm. Normally, in real situations, cars are not parked so close together and hence, this algorithm should also deliver good results for less challenging situations.

The radar image shown in Fig. 3 is the front view of a car. This is the image that is utilised as a control for the analysis

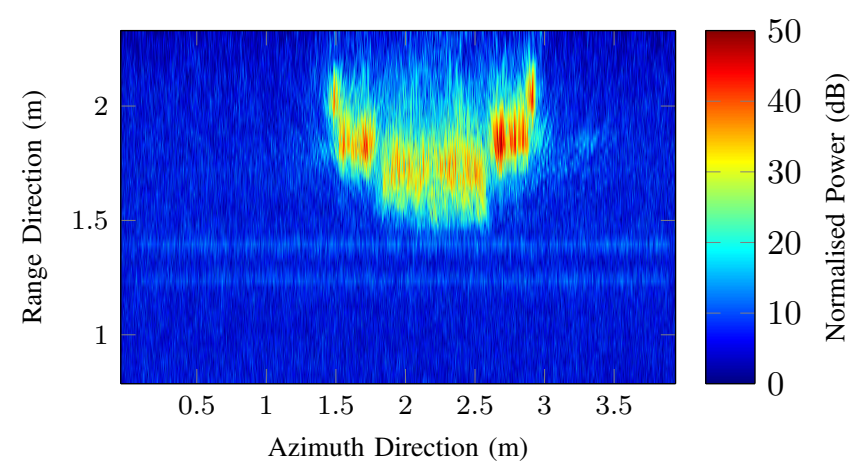

Fig. 3. Measurement result of car front view. of position errors and their effects. Here it is shown without the influence of any errors.

\section{Position ERror InVESTIGATION}

The SAR implementation so far involved a rail unit which would move in steps and in between make a radar measurement, repeating the process until the rail unit has traversed the desired distance. However this does not represent a real scenario, where a car moves constantly and the radar makes measurements with a fixed period. Also present in the real scenario are errors due to acceleration which may be too small to be detected by the acceleration sensor fixed in the car. This acceleration error influences the speed and hence the step size of the car, in other words, the distance travelled by the car in between two consecutive radar measurements.

In order to see what effect the acceleration error has on the radar images, a fictitious scenario was thought out, where it is supposed that a car with various constant starting velocities travels the same distance as the rail unit $(4.0 \mathrm{~m})$ and the radar fixed on the car makes measurements of scene with a fixed period of $200 \mu$ s. Hence the focussing part of the algorithm is tweaked such that the processing is done for radar measurements acquired not by a radar fixed on a rail unit, but rather on a car. An acceleration error is also deliberately added to produce images with position errors.

Three scenarios were tested, with the difference being the starting speeds of the car, speeds of $2.5 \mathrm{~ms}^{-1}, 5 \mathrm{~ms}^{-1}$ and $10 \mathrm{~ms}^{-1}$ were chosen. To choose a realistic value for the acceleration error, a brief online search was made and it was found that most acceleration sensors on the market have an inaccuracy of $0.0098 \mathrm{~ms}^{-2}$ (1 mg) [10], thus this was the acceleration error that was introduced in the algorithm.

\section{A. Power Analysis}

Shown in Figs. 5 and 6 are the SAR images of the same scenario as in Fig. 3 obtained from the line processing and RDA respectively. However, this time an acceleration error of $1 \mathrm{mg}$ was introduced during the processing. The starting speed

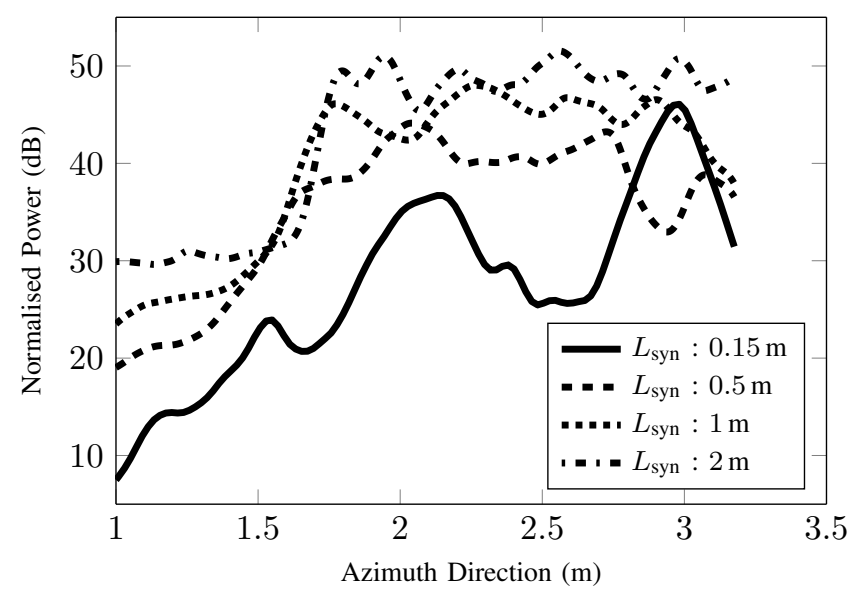

Fig. 4. Normalised power profile for a single range bin over complete azimuth length for various $L_{\text {syn }}$. 


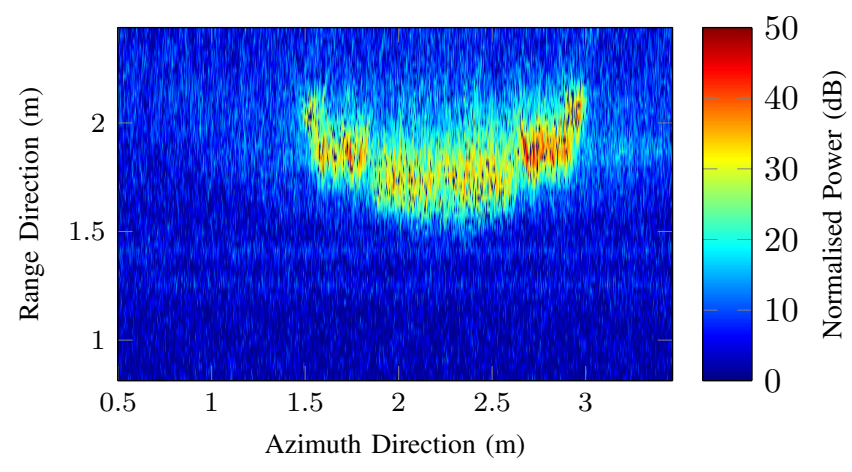

Fig. 5. Acceleration error $1 \mathrm{mg}$, starting speed $10 \mathrm{~ms}^{-1}$, obtained through line processing algorithm.

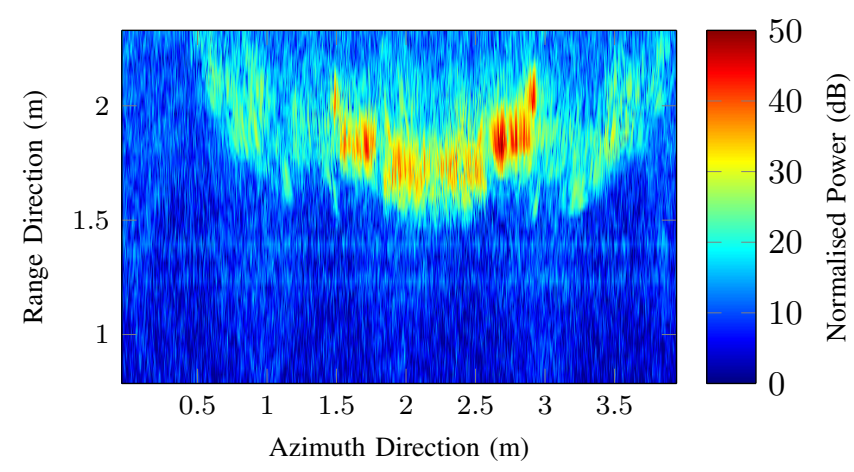

Fig. 6. Acceleration error $1 \mathrm{mg}$, starting speed $10 \mathrm{~ms}^{-1}$, obtained through RDA.

of the car was set at $10 \mathrm{~ms}^{-1}$. The most notable difference that is seen when comparing Figs. 3 and 5 is the deteriorating normalised power. This occurs because since the radar cycle is fixed at $200 \mu \mathrm{s}$ and the speed of the car increases, the distance between successive radar measurements increases. Hence for a particular synthetic aperture length, the number of radar measurements (or the number of array elements) decreases, which has a direct effect on the image quality.

In the case of Fig. 6, azimuth ambiguities are produced which show up as ghost targets in the image. This occurs due to undersampling in the azimuth direction which leads to aliasing [7]. To visualise this better, the normalised power has been plotted, in Fig. 7, for all the images of the three cases of starting speeds $\left(2.5 \mathrm{~ms}^{-1}, 5 \mathrm{~ms}^{-1}\right.$ and $\left.10 \mathrm{~ms}^{-1}\right)$ and constant acceleration error ( $1 \mathrm{mg}$ ) obtained through RDA. At a speed of $2.5 \mathrm{~ms}^{-1}$, the dynamic range is the largest and one can see the normalised power profile of the car in the azimuth and range position where it is supposed to be as shown in Fig. 3. However, when the speed is increased to $5 \mathrm{~ms}^{-1}$ and $10 \mathrm{~ms}^{-1}$ the difference between the maximum and the minimum power level decreases and it is possible to see the aliasing effects when compared to Fig. 6. The power profile confirms what the image in Fig. 6 shows, that the image obtained at higher speeds and with acceleration error is blurred and the dynamic range is reduced.
The reason why there are no azimuth ambiguities in Fig. 5 obtained through line processing can be understood when one considers the fact that the line processing algorithm functions exclusively in the time domain hence the issue of azimuth frequency undersampling does not crop up.

The undersampling is a handicap when trying to implement

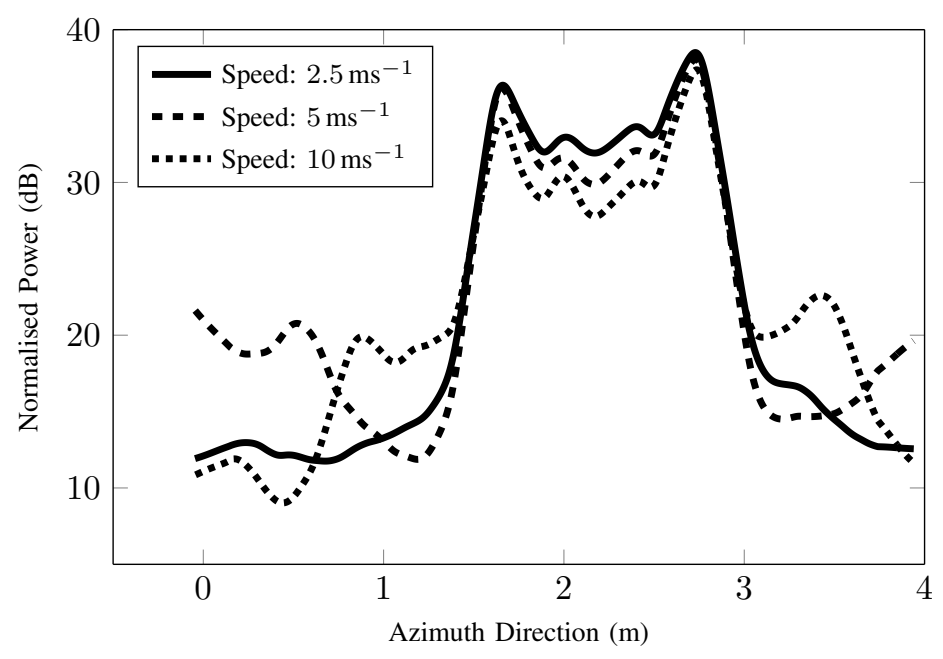

Fig. 7. Normalised power at range of $1.8 \mathrm{~m}$, starting speeds $2.5 \mathrm{~ms}^{-1}$, $5 \mathrm{~ms}^{-1}$ and $10 \mathrm{~ms}^{-1}$.

SAR for an automotive application, since the radar is only capable of making measurements with a fixed period, yet, the car may travel at a wide range of speeds leading to large step sizes (distance between two consecutive radar measurements) which leads to undersampling of the azimuth signal. A possible solution to this problem might be resampling of the radar measurements to 'generate' azimuth data at points where a measurement was missed.

\section{B. Magnitude of Acceleration Error Analysis}

Comparing the control image shown in Fig. 3 to the images with the error acceleration in Figs. 5 and 6, it is still possible to make out the contour of the car. There is not too much blurring present.

1) Line Processing Algorithm: To understand why there is no significant blurring in the image with an error acceleration, a detailed analysis of the focussing algorithm needs to be done. Focussing in the line processing algorithm is accomplished by determining the position of the array centre $(C)$ and each individual array $(E)$ element. The distance between the array centre and each individual array element is then calculated and used to focus the signal available from the radar [8]:

$$
\begin{aligned}
C= & u \cdot T_{0} \cdot\left(N+\frac{A}{2}-1\right) \\
& +\frac{1}{2} \cdot a \cdot\left[\left(\frac{A}{2}-1\right) \cdot T_{0}\right]^{2} \\
E_{\mathrm{n}}= & u \cdot T_{0} \cdot\left(N+J_{\mathrm{n}}-1\right) \\
& +\frac{1}{2} \cdot a \cdot\left[\left(J_{\mathrm{n}}-1\right) \cdot T_{0}\right]^{2} .
\end{aligned}
$$


Equations (1) and (2) represent the distance to the centre of an aperture and to each array element of the aperture respectively. $u$ is the initial speed of the car at the start of the measurement, $T_{0}$ is the radar period, $N$ is the total number of measurements made previously till the first element of the current aperture, $a$ is the acceleration error, $A$ is the number of array elements or the number of radar measurements in a single aperture and $J_{\mathrm{n}}$ is the index number which iterates over the length of the synthetic aperture, starting from one, which is the first array element to $A$ which is the last element of the aperture. Hence the distance to every single array point is calculated.

The next step in the line processing algorithm is to calculate the distance from the aperture centre to each element of the aperture. This distance is then used in subsequent steps for the phase correction of the received radar signals before they are coherently summed up [8]:

$$
\begin{aligned}
d_{\mathrm{n}}= & C-E_{\mathrm{n}} \\
= & u \cdot T_{0} \cdot\left(\frac{A}{2}-J_{\mathrm{n}}\right) \\
& +\frac{a \cdot T_{0}^{2}}{2} \cdot\left(\frac{A^{2}}{4}-J_{\mathrm{n}}^{2}\right) \\
& +a \cdot T_{0}^{2} \cdot\left(J_{\mathrm{n}}-\frac{A}{2}\right) .
\end{aligned}
$$

Equation (3) describes the terms that are left when the difference of (1) and (2) is taken. This distance between the array centre and individual array element is designated $d_{\mathrm{n}}$. It can be deduced from the result that this causes part of the acceleration error to be cancelled, hence the actual deviation due to the acceleration error is much smaller. It can also be seen that the error is largest at the end of the aperture. This occurs when $J_{\mathrm{n}}=A$. This is logical because towards the end of the aperture, the acceleration error has had the most time to be integrated, hence the value of the error is largest there. Since $A$ is the total number of array elements, it follows that the consequences of an acceleration error can be reduced when the aperture is shorter, which seems reasonable since then the error is integrated over a shorter length.

2) Range Doppler Algorithm: The core process of the range Doppler algorithm, the range cell migration correction, functions in the azimuth-frequency domain, which is dubbed as Doppler frequency. For this range correction, it is assumed that the azimuth signal is periodically sampled. However, when as in the case of a radar measurements made from an automobile which is moving with an erroneous acceleration, the azimuth signal is non-periodic, it leads to incorrect range migration correction or the correction being incomplete. The incomplete migration correction may lead to deterioration of both range and azimuth resolutions which is manifest through broadening (or blurring) of the target in the image after azimuth compression has been performed. When the migration correction is done incorrectly, the range data are not aligned but rather skewed. Hence when azimuth compression is carried out, the data in range direction experience blurring [7].
Blurring in the azimuth data occurs because of the non-optimal distribution of the azimuth bandwidth in the range cells. In the case where range migration correction is performed incorrectly (or incompletely), the azimuth signal is disseminated among different range cells and hence the azimuth bandwidth within the range cell where the target actually is located, is reduced. The azimuth bandwidth is thus divided between the range cells where the target backscattered energy is located. This results in blurring in the azimuth. However, in the case where the range migration correction is performed correctly, the backscattered energy from the target is located only in the range cell where the target is actually supposed to be and thus the complete azimuth bandwidth is exploited by the azimuth compression [7].

Nevertheless in the case of the acceleration error discussed here, the acceleration of $1 \mathrm{mg}$ is not large enough to introduce significant blurring.

\section{CONCLUSion}

It has hence been successfully shown that SAR is a viable option to fulfil the need for an imaging radar in the automotive industry. Due to the high resolution images available from the algorithm, it is possible to recognise stationary objects which can help to solve a perhaps complicated traffic situation in good time, without the use of a human driver. In this paper it has been shown that the inaccuracy of the acceleration sensors available do not handicap the utility of the SAR algorithms. However to be able to realise a functioning automotive SAR system, more efforts need to be dedicated towards generation of SAR images where the radar platform (the car) experiences sporadic, deliberate accelerations which is usually the case when cars are being driven on the roads.

\section{REFERENCES}

[1] C. Urmson, "Autonomous Driving in Urban Environments: Boss and the Urban Challenge," Journal of Field Robotics, Aug. 2008.

[2] C. Waldschmidt and H. Meinel, "Future Trends and Directions in Radar Concerning the Application for autonomous Driving," in 44th European Microwave Conference (EuMC), 2014

[3] W. Huaming and T. Zwick, "Automotive SAR for Parking Lot Detection," in German Microwave Conference, 2009.

[4] F. V. J. Mure-Dubois and D. Bonacci, "Sonar and Radar SAR Processing for Parking Lot Detection," in Radar Symposium (IRS), 2011.

[5] W. Huaming and T. Zwick, "A Novel Motion Compensation Algorithm for Automotive SAR : Simulations and Experiments," in German Microwave Conference, 2010.

[6] P. Feil and T. Chaloun, "Active Switched Antenna Array for $77 \mathrm{GHz}$ Digital Beamforming Radar," in Proceedings of the 5th European Conference on Antennas and Propagation (EUCAP), 2011.

[7] I. G. Cumming and F. H. Wong, Digital Processing of Synthetic Aperture Radar Data. Artech House, 2005.

[8] G. W. Stimson, Introduction to Airborne Radar. Scitech Publishing Inc., 1998.

[9] M. Andres, P. Feil, W. Menzel, L. Bloecher, and J Dickmann, "Analysis of Automobile Scattering Center Locations by SAR Measurements," in Radar Conference (RADAR), IEEE, 2011.

[10] Bosch, "Acceleration Sensors," Feb. 2015. [Online]. Available: https://www.bosch-sensortec.com/en/homepage/products_3/ 3_axis_sensors/acceleration_sensors/acceleration_sensors_1 